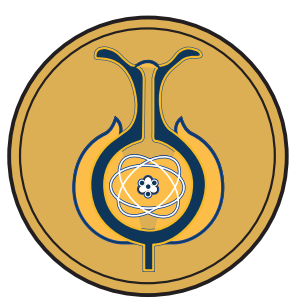

\title{
Estudio del efecto de un terremoto sobre un marco geodésico de referencia
}

Study of the effects of an earthquake on a geodetic reference frame

Estudo do efeito de um terremoto sobre um sistema geodésico de referência

\author{
Jose Valverde-Calderón \\ jose.valverde.calderon@una.cr \\ Escuela de Topografía, Catastro y Geodesia \\ Universidad Nacional \\ Heredia, Costa Rica \\ Orcid: http://orcid.org/0000-0003-3926-1761
}

Recibido-Received: 10/set/2018/Corregido-Corrected: 27/nov/2018.

Aceptado-Accepted: 8/feb/2019/Publicado-Published: 31/ene /2020.

\section{Resumen}

El objetivo de esta investigación es determinar el impacto en un conjunto de dieciséis estaciones GNSS localizadas en Costa Rica, tras el terremoto del 05 de septiembre de 2012. La investigación es de tipo explicativa y el enfoque que se utilizó es el cálculo de soluciones semanales libres, las cuales fueron luego combinadas para estimar la solución final. Basado en esta, se generaron series temporales que muestran la variación en el tiempo de la posición de las estaciones. Como resultado, se observa que las estaciones GNSS, localizadas a una distancia máxima de $140 \mathrm{~km}$ con respecto al epicentro del evento, sufrieron cambios tanto en su ubicación como en la dirección del movimiento y en la magnitud de su velocidad, siendo las estaciones ubicadas en la península de Nicoya las que presentan los mayores cambios. Se concluye que el evento estudiado modificó de forma significativa la posición de trece de las estaciones analizadas, mientras que tres no fueron afectadas.

Palabras claves: Geodesia; terremoto; deformación; GNSS

\section{Abstract}

The principal objective of this research is to analyze the impact of the earthquake of September 05, 2012 on a set of sixteen Global Navigation Satellite System (GNSS) stations located in Costa Rica. This is an explanatory investigation based on the calculation of weekly free solutions, which were later combined to obtain a final solution which was in turn used to generate time series that show variations in the position of the stations. It was observed that GNSS stations located at a maximum distance of $140 \mathrm{~km}$ from the epicenter of the earthquake experienced changes in both their position and in the magnitude of their 
speed, with the stations located in the Peninsula de Nicoya being the most affected. It is concluded that the event studied produced significant changes in the position of thirteen of the analyzed stations, while three stations were not affected by the earthquake.

Keywords: Geodesy; earthquake; deformation; GNSS

\section{Resumo}

0 principal objetivo desta pesquisa é determinar o impacto sobre um conjunto de dezesseis estações GNSS localizados na Costa Rica, após o terramoto de 05 de setembro de 2012. A investigação é do tipo explicativa e o enfoque utilizado é o cálculo de soluções semanais livre, que foram então combinados para estimar a solução final. Com base nisso, foram geradas séries temporais que mostram a variação no tempo da posição das estações. Como resultado, observa-se que as estações GNSS, localizadas a uma distância máxima de 140 km em relação ao epicentro do evento, sofreram alterações tanto em sua localização quanto na direção de movimento e na magnitude de sua velocidade, sendo as estações localizadas na península de Nicoya as que apresentam as maiores alterações. Conclui-se que o evento estudado modificou significativamente a posição de treze das estações analisadas, enquanto três não foram afetadas.

\section{Palavras-chave: Geodésia; Terremoto Deformação; GNSS}

\section{Introducción}

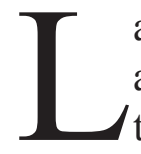
a información geoespacial es, en la actualidad, un elemento fundamental para las actividades humanas de la civilización moderna (Ramírez \& Vargas, 2014). Información para la planificación urbana, gestión de riesgos, optimización de las áreas dedicadas a uso agrícola y en general cualquier actividad económica, requiere de datos del entorno. La información geoespacial puede ser generada de varias formas: métodos topográficos convencionales, técnicas fotogramétricas, uso de escáneres, entre otros. Sin embargo, es un requerimiento actual que los datos estén referidos a un mismo sistema de coordenadas, de forma tal que estos sean compatibles e interoperables y es en ese aspecto donde tienen un gran aporte los métodos geodésicos.

La geodesia clásica costarricense utilizó el datum de Ocotepeque, establecido en 1934. La materialización de este se dio mediante un mojón en el cual se hicieron cumplir por definición las condiciones dadas en la época para el establecimiento del datum geodésico, basándose en el elipsoide de revolución calculado por Clarke en el año 1866 (Programa de Regularización de Catastro y Registro de Costa Rica, 2007).

A través de una red GPS con cobertura nacional, la cual fue medida a inicios de los años 90 por el Catastro Nacional, se dio la materialización del sistema CRTM90. En el año 1998, mediante un proyecto financiado por RECOPE, se crea una nueva proyección para el país conocida como Costa Rica Transversal de Mercator de 1998 (Programa de Regularización de Catastro y Registro de Costa Rica, 2007).

En el marco de ejecución del Programa de Regularización de Catastro y Registro (PRCR), creado mediante la ley 8154 de 2001 y para satisfacer la necesidad de tener un sistema geodésico de referencia acorde con las metodologías y técnicas usadas en la actualidad, se estableció el sistema de referencia CR05, al cual se le 
asocia la proyección cartográfica denominada CRTM05. El marco geodésico CR05 está definido por la red de primer orden, la cual nominalmente está conformada por 34 vértices geodésicos; la medición de dicha red se realizó entre agosto y octubre de 2005. Desde mayo de 2010, funciona la red de estaciones de operación continua oficial del país, la cual está conformada por 8 estaciones (Programa de Regularización de Catastro y Registro de Costa Rica, 2007).

El surgimiento del GPS se da a partir del sistema TRANSIT, el cual presentó limitaciones desde sus inicios, por lo que como una evolución del sistema TRANSIT se desarrolla el NAVSTAR-GPS (NAVigation Satellite Time and Ranging- Global Positioning System, con su traducción en inglés) (Ostini, 2012). El GPS es un sistema que permite la radionavegación, basado en la señal transmitida por satélites, lo que permite a los usuarios, determinar su posición tridimensional, velocidad y tiempo, las 24 horas del día, en cualquier parte del planeta (Hofmann- Wellenhof \& Moritz, 2005).

En la geodesia moderna el uso del GPS es muy importante ya que permite la definición y mantenimiento de un marco geodésico global, el estudio de la cinemática del este, así como establecer los marcos continentales y nacionales. Además, es utilizado en la definición de redes pasivas (mojones) y redes activas (EMC).

Las coordenadas que definen un marco geodésico de referencia son determinadas para una época de referencia; por lo que, para conocer la posición en cualquier otra, se deben actualizar estos valores en la medición, tomando en cuenta la aplicación para la cual se requieren las coordenadas, la exactitud buscada y la escala del producto cartográfico a generar (si fuera el caso).
Debido a la ubicación geográfica de Costa Rica y por la interacción de las placas Cocos y Caribe, el país es muy propenso a ser afectado por terremotos de magnitud importante, como el ocurrido el 05 de septiembre de 2012, con una magnitud 7,6 Mw. En consecuencia de este evento, se produjeron cambios en la topografía del terreno en las áreas costeras entre ellas Sámara, Garza (zonas más próximas al epicentro).

\section{Marco teórico}

\section{Definición de geodesia}

La geodesia es la ciencia que se encarga de estudiar la forma y dimensiones de la Tierra, así como su campo de gravedad (Torge, 2001). Los problemas básicos que debe resolver, de acuerdo con Seeber (2003) son:

1) La determinación de posiciones tridimensionales precisas a una escala global, regional y local (establecimiento de control geodésico para aplicaciones científicas e ingeniería)

2) Determinación del campo de gravedad de la Tierra y las funciones lineales de este campo (un geoide preciso y modelos de geopotencial)

3) Medición y modelado de fenómenos geodinámicos (variaciones del movimiento del polo y rotación de la Tierra, deformaciones de la corteza, estudios atmosféricos, entre otros).

\section{Sistemas y marcos geodésicos de referencia}

La geodesia requiere de marcos de referencia consistentes, exactos y confiables para la medición y mapeo de la superficie de la Tierra y sus variaciones en el tiempo. Los marcos geodésicos de referencia son la base 
para gran cantidad de aplicaciones prácticas tales como redes nacionales que sustentan la cartografía de un país, la navegación precisa, el mantenimiento de sistemas de información geoespacial, así también como para aplicaciones científicas relacionadas con el estudio de fenómenos de naturaleza geofísica que ocurren en la Tierra. (Angermann et al., 2004).

Un sistema de referencia es la definición conceptual completa de como un sistema coordenado está formado. Define el origen y orientación de los planos fundamentales o ejes de este. También incluye los modelos físicos y matemáticos fundamentales. Se denominan convencionales cuando los modelos, constantes numéricas y algoritmos son especificados (Seeber, 2003).

Un Sistema de Referencia Terrestre (TRS, por sus siglas en inglés) es un sistema de referencia espacial, que rota con la Tierra en su movimiento diario en el espacio. En él las posiciones de las estaciones de observación están vinculadas a la superficie sólida de la Tierra con coordenadas, las cuales presentan variaciones en el tiempo debido a efectos geofísicos (deformación por tectónica o mareas) (Petit \& Luzum, 2010).

Un marco de referencia es la realización práctica del sistema de referencia a través de observaciones. El Marco de Referencia Terrestre (TRF, por sus siglas en inglés) es elaborado al usar observaciones de la geodesia espacial y es accesible, para los usuarios, a través de valores numéricos, las cuales consisten en posiciones como una función del tiempo de una red de estaciones geodésicas. El TRF es la realización del Sistema de Referencia Terrestre (TRS) (Altamimi, Collilieux \& Métivier, 2011).

\section{Posición instantánea de una esta- ción GPS}

En las convenciones del Servicio Internacional para la Rotación de la Tierra y los Sistemas de Referencia (IERS, por sus siglas en inglés) es recomendado que la posición instantánea de una estación, la cual está fija a la corteza de la Tierra, sea descrita por la posición de la estación regularizada y por modelos de corrección convencionales. Las realizaciones actuales del ITRF usan una posición de las estaciones en una época de referencia y a una velocidad constante para describir el movimiento de la estación en el tiempo (Bloßfeld, Seitz \& Angermann, 2014).

\section{Fundamentos de ajuste secuencial y técnica de combinación}

Al igual que otras ciencias, datos de observaciones son usados en la geodesia para hacer inferencia de la realidad física. Con el fin de obtener información sobre el mundo real, se trata de capturar el conglomerado de la realidad física por medio de un modelo matemático. Este consta de 2 partes: la funcional y la estocástica. Mediante el modelo funcional, se describe la relación que hay entre los propios observables o entre los observables y los parámetros desconocidos. El modelo estocástico es usado para considerar la incertidumbre o la variabilidad de los datos empíricos (Teunissen, 1998).

La necesidad del ajuste de observaciones o la estimación de parámetros surge, usualmente, cuando se tiene que resolver alguna inconsistencia en el sistema de ecuaciones, la cual, en geodesia, es redundante (hay más observaciones que incógnitas). El principio de ajuste usado con frecuencia es el de mínimos cuadrados. Este algoritmo es fácil de aplicar y tiene importantes 
propiedades de optimización. La estimación por mínimos cuadrados es equivalente a la estimación máxima de probabilidad (en caso de que los observables sigan una distribución normal) y en el caso lineal, también es equivalente a la mejor estimación lineal no-sesgada (Teunissen, 1998).

En la geodesia clásica, cuando se medían las redes de triangulación o trilateración y antes del desarrollo de computadoras, con suficiente capacidad para procesar gran cantidad de observaciones, era común que las redes geodésicas se procesaran en bloques y posteriormente, las soluciones de los bloques individuales eran combinadas para obtener la solución global de la red. A este proceso se le conoce como "estimación secuencial por mínimos cuadrados" o LSE, por sus siglas en inglés (Brockmann, 1997).

Por ejemplo, una estación GPS que efectué un registro cada $30 \mathrm{~s}$ de 4 satélites, recolecta durante un día (24 horas de observación) 11520 observaciones. Por tanto, en una semana esta estación recolecta 80640 observaciones. Considerando que hay redes conformadas por varias decenas de estaciones GPS y que estas estaciones comúnmente recolectan observaciones por años, se concluye que un procesamiento en el nivel de observaciones, de toda esta información, en términos computacionales no es viable (Seitz, 2014).

La combinación de diferentes técnicas geodésicas es un procedimiento común con el fin de calcular productos geodésicos. $\mathrm{La}$ combinación de observaciones de la geodesia espacial se ha convertido en una herramienta fundamental para el cálculo de parámetros en el sistema Tierra, así como para la realización de los sistemas de referencia. Precisión, exactitud, estabilidad a largo plazo y fiabilidad de los productos pueden ser mejorados mediante la combinación de diferentes técnicas de observación, que proporcionan una sensibilidad individual con respecto a ciertos parámetros (Seitz, 2014).

La combinación de los datos de diferentes técnicas de observación se puede realizar en tres niveles: en el nivel de observaciones, en ecuaciones normales y en parámetros. El funcionamiento básico en el proceso de combinación de ecuaciones normales es el stacking de las mismas. Esto significa que los parámetros comunes a más de una ecuación normal son estimados en un único valor: el mismo parámetro contenido en al menos dos sistemas de ecuaciones normales de entrada es compuesto en un solo parámetro tras la combinación de las ecuaciones normales. Este procedimiento es conocido también como "Bloque de Helmert" (Thaller, 2008).

\section{Discontinuidades en series de tiempo}

Los Sistemas de Navegación Global por Satélite (GNSS, por sus siglas en inglés) son una fuente de información importante para lograr una mejor comprensión de los fenómenos geofísicos y climáticos. Sin embargo, las discontinuidades en las series de tiempo pueden ser un factor limitante para la estimación de las tendencias a largo plazo (Bruni, 2014).

Las técnicas GNSS ofrecen, hoy en día, una contribución al monitoreo de la forma de la Tierra, su cinemática y las variaciones en diferentes escalas temporales y espaciales. Si las discontinuidades no son debidamente contabilizadas y removidas, estas podrían afectar el modelado confiable de las tendencias de largo período y de las variaciones no lineales, originadas por fenómenos relacionados con cambios geofísicos y climáticos (Bruni, 2014).

Las discontinuidades se producen en casi todas las series de tiempo de coordenadas derivadas de datos GNSS (Williams, 
2008). Muchas de ellas son causadas por actualizaciones de modelos y cambios en las estrategias de procesamiento, pero también puede haber otras fuentes. Los terremotos, por ejemplo, pueden causar discontinuidades y cambios de velocidad de una estacion cerca del epicentro. Otras pueden estar asociadas con cambios en el equipo (por ejemplo, debido a la sustitución de antenas o receptores, actualizaciones de firmware, etc). Estas también pueden deberse a una razón desconocida, por ejemplo, el mal funcionamiento de los equipos, vandalismo, errores humanos, entre otros (Ostini, 2012).

En la geodesia clásica, las coordenadas de las estaciones eran constantes con el tiempo. Las coordenadas estáticas no solo son más adecuadas para una Tierra estática, sino que también sirven en una Tierra dinámica en la cual los métodos de posicionamiento existentes carecían de la precisión para detectar movimientos tectónicos durante largos periodos de tiempo. Con el desarrollo de técnicas de posicionamiento por satélite, los movimientos de las placas se pudieron determinar más fácilmente con periodos más cortos de observación. El sistema de referencia concebible más simple para una Tierra dinámica utiliza un Modelo de Velocidad Constante (CVM, por sus siglas en inglés) en el que, las coordenadas cartesianas geocéntricas $(\mathrm{X}, \mathrm{Y}$, Z) para cada estación geodésica se expresan como una función lineal de tiempo (Bevis \& Brown, 2014).

Los modelos de trayectoria modernos en geodesia se componen de tres submodelos que representan las tendencias seculares, oscilaciones anuales y saltos instantáneos. Tradicionalmente el modelo de tendencia invoca una velocidad constante de la estación. Esto se puede generalizar al asumir que la posición es una función polinómica en el tiempo. El modelo de trayectoria también se puede aumentar según sea necesario, mediante la inclusión de una o más funciones logarítmicas, con el fin de considerar los patrones típicos de movimiento postsísmica. En este caso, se hace referencia a modelos puramente cinemáticos. Para los geodestas, estos modelos son útiles porque proporcionan un medio sencillo y compacto para predecir la posición de una determinada estación en día determinado. Los geofísicos están, generalmente, más preocupados por las trayectorias de las estaciones debido a la información que proveen sobre el comportamiento dinámico de la Tierra sólida (Bevis \& Brown, 2014).

\section{Metodología}

\section{Selección de las estaciones en Costa Rica}

El principal interés del proyecto es analizar el efecto del evento del 05 de septiembre de 2012 sobre la infraestructura geodésica de Costa Rica, por tanto, se procedió a identificar cuales estaciones GPS se podrían considerar para el procesamiento. En el país varias instituciones han establecido y mantienen redes GPS:

1) La red oficial del país, administrada por el Instituto Geográfico Nacional (IGN)

2) La red establecida por el Instituto Costarricense de Electricidad

3) La red establecida por el Observatorio Vulcanológico y Sismológico de Costa Rica

4) La red establecida por UNAVCO

5) Otras estaciones que son producto de esfuerzos de otros entes, como la estación de la ETCG-UNA, la del Instituto Costarricense de Acueductos y Alcantarillados, entre otras 
Luego de analizar la disponibilidad y la forma de acceso a los datos, así como la distribución geográfica, se seleccionó una red que está conformada por 16 estaciones, cuya distribución espacial se muestra en la Figura 1. En la Tabla 1 se indica el nombre de las estaciones seleccionadas y el cantón donde se ubican.

Tabla 1

Estaciones en Costa Rica usadas en el procesamiento

\begin{tabular}{llll}
\hline \multicolumn{1}{c}{ Estación } & \multicolumn{1}{c}{ Cantón } & \multicolumn{1}{c}{ Estación } & \multicolumn{1}{c}{ Cantón } \\
\hline BON2 & Puntarenas & NEIL & Corredores \\
CIQU & San Carlos & NICY & Nicoya \\
ETCG & San Pablo & PNE2 & Santa Cruz \\
GRZA & Nicoya & PUNT & Puntarenas \\
HUA2 & Santa Cruz & RIDC & San Jose \\
LAFE & Puntarenas & SAGE & San Isidro de El General \\
LIBE & Liberia & SAJU & Santa Cruz \\
LIMN & Limón & VERA & San Carlos \\
\hline
\end{tabular}

Nota: fuente propia de la investigación.

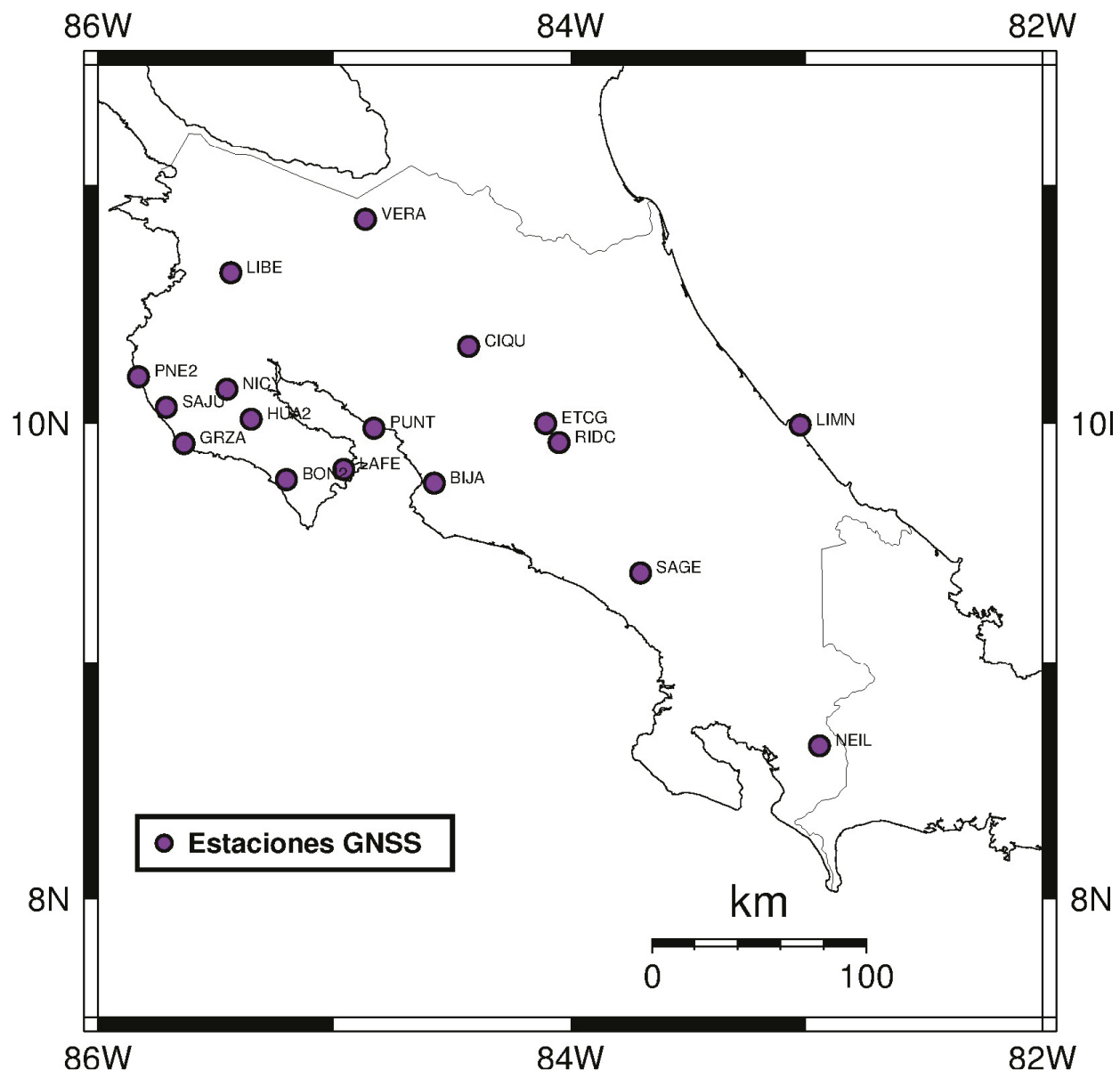

Figura 1. Ubicación de las estaciones en Costa Rica. Fuente: Elaboración propia, tomando como base la información del programa GMT 


\section{Selección de las estaciones de referencia}

Es necesario considerar en el procesamiento estaciones externas al área de estudio, a efectos de que luego, algunas de estas estaciones sean utilizadas como referencia al momento de efectuar la combinación de las soluciones libres.

Tomando en cuenta las capacidades del programa Bernese para el procesamiento de líneas bases largas, de hasta $2000 \mathrm{~km}$ al utilizar la estrategia QIF para resolver ambigüedades, se seleccionaron estaciones principalmente del Servicio GNSS Internacional (IGS), cuyos datos están disponibles de forma gratuita, pero, además, son procesadas tanto por el IGS como por el Sistema de Referencia Geocéntrico para las Américas (SIRGAS), por lo que se conoce también la velocidad de cada estación.

La red definida consta de 24 estaciones externas y su ubicación espacial se puede ver en la Figura 2.

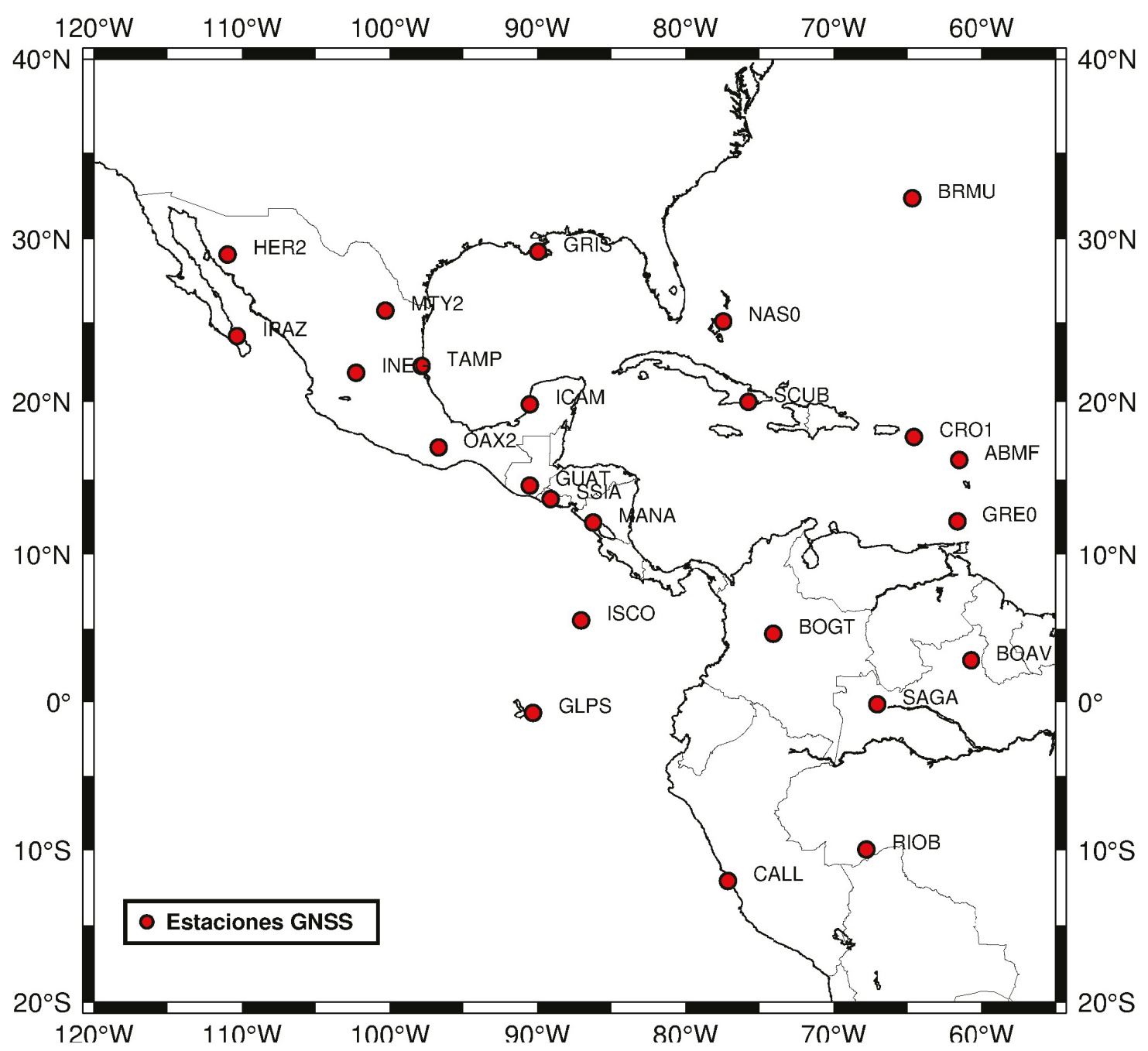

Figura 2. Ubicación de las estaciones externas. Fuente: Elaboración propia, tomando como base la información del programa GMT 


\section{Procesamiento de los datos}

Una vez definidas las estaciones a procesar, se procede a la descarga de los datos de observación en formato RINEX. El procesamiento de las observaciones se realizó en el programa Bernese versión 5.2, con la licencia disponible en el Centro Nacional de Procesamiento de Datos GNSS (CNPDG) de la UNA. Como requerimiento para el procesamiento, es necesario disponer de información que indique la trayectoria verdadera que siguieron los satélites. Para este fin, se utilizaron las efemérides precisas calculadas y publicadas por el IGS. También, se descargaron los Parámetros de Orientación de la Tierra (EOP, por sus siglas en inglés), los cuales son necesarios para el cálculo de las órbitas en el sistema inercial celeste.

Relacionado con información para el modelado a priori de la troposfera, se utilizó la aplicación vmf2grd.bat, escrita por el Dr. Jorge Moya Zamora, del CNPDG, para la descarga de los datos con los coeficientes de las mallas globales, las cuales son aplicadas con la Función de Mapeo de Viena (VMF por sus siglas en inglés).

Una vez descargados los datos de observación, los cuales están en formato de compresión de Hatanaka, se convirtieron a formato RINEX 2.11 y se utilizó una rutina escrita en el lenguaje PERL por el Dr. Mauricio Gende, de la Universidad de La Plata, la cual elimina las observaciones GLONASS y ordena la estructura de los archivos.

Finalmente, es necesario generar un conjunto de archivos requeridos para el procesamiento, los cuales son:

1) Archivo de abreviaturas, requerido por Bernese para identificar las líneas base

2) Un archivo con la información del tipo receptor y de antena en cada estación, así como los cambios en el equipo
3) Un archivo con coordenadas aproximadas de las estaciones

4) Un archivo con las velocidades de las estaciones, requerido para la actualización de las coordenadas aproximadas a la época de medición

5) El archivo con los coeficientes para modelar el efecto de la carga oceánica

6) El archivo con los coeficientes para modelar el efecto de la carga atmosférica

Las observaciones fueron procesadas por semana, de forma que, para cada procesamiento semanal, se obtuvo una solución diaria libre y una combinada correspondiente a ella. La solución septenaria es el insumo utilizado para la combinación. El periodo de proceso considerado es del 16 de enero de 2011 al 07 de enero de 2017, para un total de 312 semanas, de forma que en la combinación se consideraron 312 soluciones libres.

\section{Combinación de las soluciones libres}

Para efectuar la combinación de las soluciones libres, las cuales proveerán como resultado las velocidades de las estaciones, las series de residuales y las series con las coordenadas de las estaciones, es necesario definir un conjunto de estaciones de referencia para determinar el datum.

Como se comentó, la red externa definida para el proyecto consta de 24 estaciones. De estas, era necesario definir algunas, para ser usadas como estaciones fiduciales. Para este fin, se consideró que las estaciones de referencia óptimas son aquellas que han sido procesadas de forma continua, por lo que se conoce valores de velocidad, no presentan discontinuidades en la serie temporal y tienen la mayor cantidad de semanas con soluciones de procesamiento realizado. 
Luego del análisis, según los criterios anteriormente establecidos, se seleccionaron 7 estaciones para ser usadas como fiduciales: TAMP, ICAM, MANA, CRO1, BOGT, BOAV y CALL. En la Figura 3 se muestra la ubicación de estas estaciones.

Durante el procesamiento se pueden presentar circunstancias ajenas al control de quien está procesando los datos; por ejemplo, las estaciones pueden dejar de registrar observaciones o se pueden producir cambios en los equipos instalados en ellas (antena, receptor o ambos). Por ende, es necesario considerar las posibles modificaciones en sus configuraciones. Es por esta razón, que como resultado del procesamiento semanal, se llevó un registro de aquellas inconsistencias encontradas por el programa al momento de realizar el cálculo de los parámetros de interés. En la Figura 4 se muestra un ejemplo.

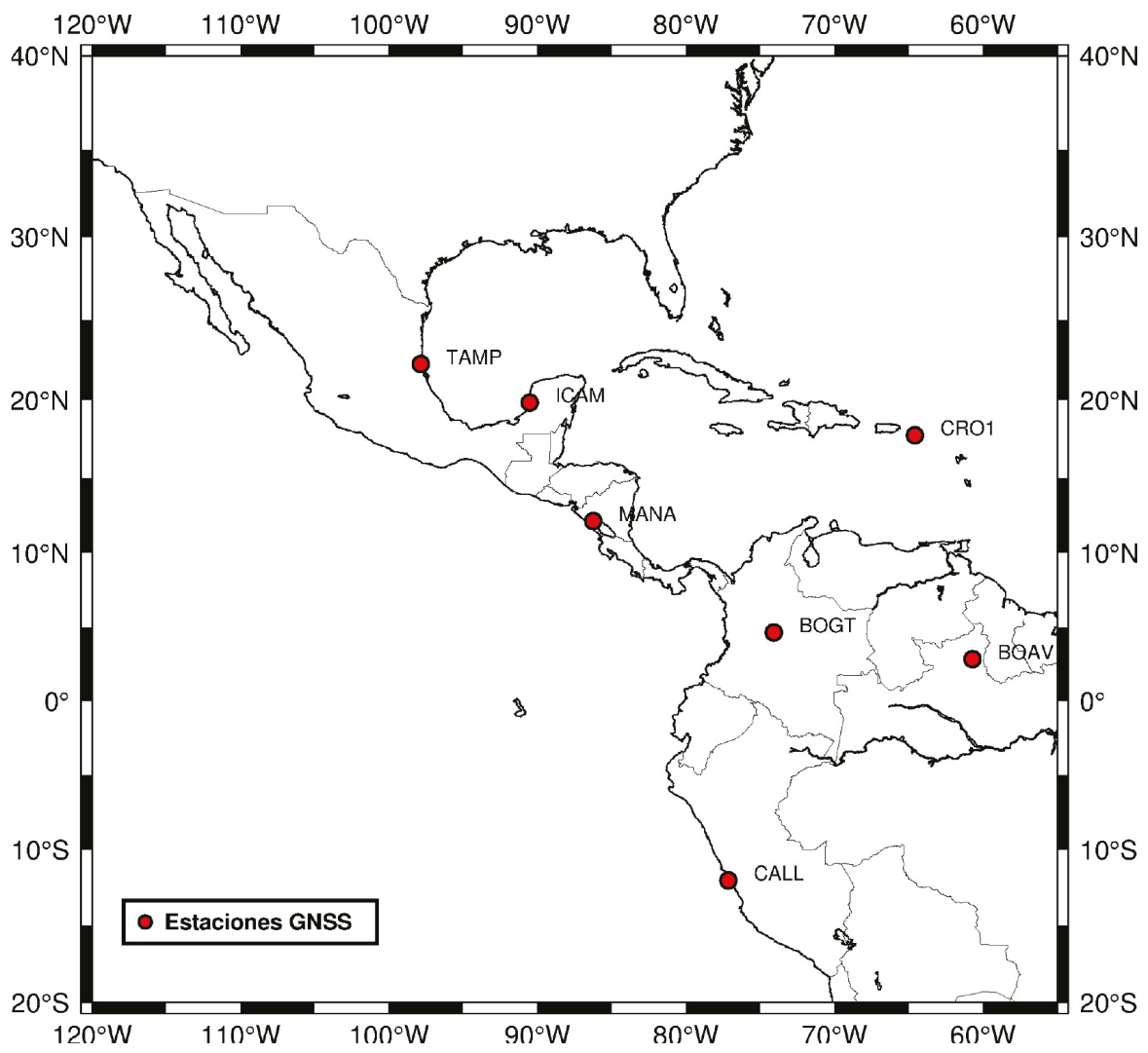

Figura 3. Ubicación de las estaciones fiduciales.

Fuente: Elaboración propia, tomando como base la información del programa GMT

\begin{tabular}{|c|c|c|c|c|c|}
\hline Station & First obs. epoch & Last obs. epoch & Receiver type & Antenna type & dome \\
\hline GLPS 42005M002 & $2012-12-02 \quad 00: 00: 00$ & $2012-12-05 \quad 23: 59: 30$ & ASHTECH UZ-12 & ASH701945B_M & NONE \\
\hline GLPS 42005M002 & $2012-12-07 \quad 00: 00: 00$ & $2012-12-08 \quad 23: 59: 30$ & ASHTECH UZ-12 & ASH701945B M & SCIT \\
\hline
\end{tabular}

Figura 4. Ejemplo de una inconsistencia en el procesamiento.

Fuente: Elaboración propia. 
Se considera una inconsistencia aquella diferencia entre la información contenida en la cabecera del archivo de observación y el archivo con la información de las estaciones. Cuando el programa identificó alguna inconsistencia, se realizó una revisión manual a efectos de determinar su significancia. En caso de determinar que fue un error durante el procesamiento, no se calculó de nuevo la semana, sino que la estación que dio problemas fue eliminada de la combinación para el periodo que comprende la inconsistencia.

Finalmente, la combinación fue realizada tomando como referencia la solución SIR15P01 (Sánchez \& Drewes, 2016), y se consideró como época de la combinación la 2014.0. Esto es importante, por cuanto las velocidades calculadas estas referidas, específicamente a dicha época.

\section{Elaboración de las series temporales}

Luego de realizada la combinación, se obtiene una serie de productos: coordenadas y velocidades dadas a la época de referencia, residuales, los cuales muestran la diferencia entre las soluciones semanales individuales y la solución combinada que considera un modelo lineal y la solución final para cada semana procesada.

Uno de los intereses del proyecto fue determinar el comportamiento cinemático de las estaciones luego del evento del 05 de septiembre de 2012, razón por la cual es necesario generar las series temporales de las estaciones, que muestran, de manera gráfica, el comportamiento de la estación para el periodo considerado.

En la sección de resultados se muestran las gráficas de algunas de las estaciones procesadas. Dichas gráficas fueron realizadas a partir del archivo producto de la combinación, el cual contiene la solución semanal final. Esta información fue extraída para cada estación de interés a partir de una rutina generada por el Sr. Jean Carlo Arroyo Ruiz y el Máster Carlos Sevilla Hernández, estudiante y docente de la ETCG, respectivamente.

\section{Resultados}

A efectos de presentar los resultados de coordenadas y velocidades, estos se han dividido en dos: los valores para antes y para después del evento del 05 de septiembre de 2012 .

Coordenadas de las estaciones y velocidades para el periodo comprendido del 16 de enero de 2011 al 04 de septiembre de 2012

En la Tabla 2 se muestran los valores de las coordenadas y las velocidades geocéntricas para el periodo comprendido entre el 16 de enero de 2011 y el 04 de septiembre de 2012: 
Tabla 2

Coordenadas y velocidades de las estaciones costarricense válidas para antes del evento del 05 de septiembre de 2012, época 2014.0

\begin{tabular}{lcccccc}
\hline & \multicolumn{3}{c}{ Coordenadas geocéntricas } & \multicolumn{3}{c}{ Velocidades geocéntricas } \\
\multicolumn{1}{c}{ Estación } & $\mathbf{X}(\mathbf{m})$ & $\mathbf{Y}(\mathbf{m})$ & $\mathbf{Z}(\mathbf{m})$ & $\mathbf{v X}(\mathbf{m m} / \mathbf{y r})$ & $\mathbf{v Y}(\mathbf{m m} / \mathbf{y r})$ & $\mathbf{v Z}(\mathbf{m m} / \mathbf{y r})$ \\
\hline BON2 & 525755.71208 & -6264346.20257 & 1074595.46265 & 12.2 & 5.5 & 19.8 \\
CIQU & 609048.99260 & -6246628.64007 & 1135475.59855 & 15.6 & 3.3 & 11.1 \\
ETCG & 645208.33188 & -6249842.13379 & 1100399.59331 & 11.2 & 5.96 & 16.9 \\
GRZA & 478171.97485 & -6265306.42437 & 1091052.76103 & 18.7 & 18.7 & 24.0 \\
HUA2 & 509098.42856 & -6261454.41514 & 1102283.53869 & 16.9 & 11.7 & 21.6 \\
LAFE & 552167.24684 & -6261307.49363 & 1079237.40067 & 10.9 & 10.6 & 20.0 \\
LIBE & 498678.71658 & -6249679.81823 & 1168903.30920 & 10.9 & 8.6 & 20.4 \\
LIMN & 762717.26068 & -6235556.50477 & 1099500.41224 & 14.7 & 9.4 & 7.6 \\
NEIL & 774610.26362 & -6258471.54582 & 952302.99395 & 21.9 & 10.7 & 21.9 \\
NICY & 497631.07233 & -6259516.73896 & 1115789.96936 & 14.3 & 9.3 & 24.4 \\
PNE2 & 456660.20024 & -6261476.80226 & 1121504.62575 & 11.1 & 8.4 & 21.7 \\
PUNT & 565870.42074 & -6256745.16260 & 1098060.89783 & 11.9 & 6.4 & 19.5 \\
RIDC & 651566.60002 & -6250735.95469 & 1091707.74224 & 11.8 & 2.2 & 16.4 \\
SAGE & 690230.80540 & -6256292.39590 & 1032020.61026 & 22.0 & 7.8 & 21.5 \\
SAJU & 469761.66016 & -6263058.94775 & 1107572.41260 & 17.8 & 15.7 & 22.1 \\
VERA & 560280.94580 & -6239744.22662 & 1193120.02255 & 16.4 & 0.3 & 8.5 \\
\hline
\end{tabular}

Nota: fuente propia de la investigación.

\section{Tabla 3}

Coordenadas y velocidades de las estaciones costarricense válidas para después del evento del 05 de septiembre de 2012, época 2014.0

\begin{tabular}{lcccccc}
\hline \multicolumn{1}{c}{ Estación } & $\mathbf{X}(\mathbf{m})$ & $\mathbf{Y}(\mathbf{m})$ & $\mathbf{Z}(\mathbf{m})$ & $\mathbf{v X}(\mathbf{m m} / \mathbf{y r})$ & $\mathbf{v Y}(\mathbf{m m} / \mathbf{y r})$ & $\mathbf{v Z}(\mathbf{m m} / \mathbf{y r})$ \\
\hline BON2 & 525755.64833 & -6264346.53207 & 1074595.11878 & 1.4 & 9.9 & 2.6 \\
CIQU & 609048.90882 & -6246628.64647 & 1135475.54856 & 9.0 & 1.6 & 14.1 \\
ETCG & 645208.29709 & -6249842.13613 & 1100399.57911 & 7.5 & 5.1 & 14.8 \\
GRZA & 478171.48100 & -6265307.12013 & 1091052.15154 & 4.2 & -4.3 & -6.2 \\
HUA2 & 509098.21377 & -6261454.70049 & 1102283.16932 & -5.2 & 3.8 & -10.6 \\
LAFE & 552167.17252 & -6261307.58605 & 1079237.25756 & 4.6 & 1.3 & 5.8 \\
LIBE & 498678.61680 & -6249679.83561 & 1168903.03353 & 0.3 & -0.1 & 2.8 \\
LIMN & 762717.26701 & -6235556.50466 & 1099500.41897 & 15.6 & 8.8 & 6.1 \\
NEIL & 774610.26511 & -6258471.55038 & 952302.99642 & 20.7 & 10.1 & 21.8 \\
NICY & 497630.82580 & -6259516.89852 & 1115789.52538 & -7.3 & -3.9 & -10.6 \\
PNE2 & 456659.91254 & -6261477.07781 & 1121504.41792 & -2.1 & -1.4 & -9.2 \\
PUNT & 565870.29483 & -6256745.18970 & 1098060.78802 & 5.2 & 1.4 & 6.7 \\
RIDC & 651566.57211 & -6250735.94932 & 1091707.73234 & 8.7 & 4.2 & 15.6 \\
SAGE & 690230.80091 & -6256292.39579 & 1032020.61255 & 20.6 & 7.7 & 22.5 \\
SAJU & 469761.24285 & -6263059.51833 & 1107572.03645 & 0.1 & 1.9 & -11.1 \\
VERA & 560280.86368 & -6239744.24292 & 1193119.90894 & 5.9 & 0.9 & -0.5
\end{tabular}

Nota: fuente propia de la investigación. 
Con respecto a los resultados mostrados en la Tabla 2, se aclara que los valores son válidos únicamente para el periodo indicado y que no deben utilizarse en periodos distintos a este.

\section{Coordenadas de las estaciones y ve- locidades Periodo: del 06 de septiem- bre de 2012 al 07 de enero de 2017}

En la Tabla 3 se muestran los valores de las coordenadas y las velocidades geocéntricas para el periodo comprendido entre el 16 de enero de 2011 y el 04 de septiembre de 2012:

\section{Valores de los desplazamientos ho- rizontales y verticales}

Una vez hecha la combinación de las soluciones libres, se cuenta con dos conjuntos de coordenadas para las estaciones analizadas (coordenadas antes y después del terremoto de 05 de septiembre de 2018). Con estos conjuntos, se procedió a determinar los vectores de desplazamiento tanto horizontal como vertical. Se aclara que los valores de desplazamiento vertical corresponden a diferencias de altura elipsoidal mientras que, para el cálculo de los vectores horizontales, las coordenadas geocéntricas fueron convertidas a coordenadas geodésicas y luego, a coordenadas de cuadrícula en la proyección CRTM05. Los valores de los desplazamientos se muestran en la Tabla 4. En la Figura 5 se representa gráficamente los vectores de desplazamiento horizontal.

Tabla 4

Valores de los desplazamientos horizontales y verticales en las estaciones costarricenses

\begin{tabular}{cccc}
\hline Estación & $\begin{array}{c}\text { Desplazamiento } \\
\text { vertical }(\mathbf{m m})\end{array}$ & $\begin{array}{c}\text { Desplazamiento } \\
\text { horizontal }(\mathbf{m m})\end{array}$ & $\begin{array}{c}\text { Azimut del desplazamiento } \\
\text { horizontal (grados) }\end{array}$ \\
\hline CIQU & -8.0 & 60.0 & 241.0 \\
ETCG & -2.0 & 22.0 & 252.0 \\
GRZA & 456 & 682.0 & 220.0 \\
LAFE & 7.0 & 88.0 & 204.0 \\
LIBE & -46.0 & 208.0 & 199.0 \\
NEIL & -1.0 & 3.0 & 8.0 \\
NICY & 47.0 & 340.0 & 208.0 \\
PUNT & -23.0 & 105.0 & 237.0 \\
RIDC & 3.0 & 18.0 & 257.0 \\
SAGE & 2.0 & 3.0 & 125.0 \\
SAJU & 441.0 & 477.0 & 232.0 \\
VERA & -8.0 & 90.0 & 216.0 \\
BON2 & 254.0 & 268.0 & 183.0 \\
HUA2 & 196.0 & 264.0 & 206.0 \\
LIMN & 1.0 & 9.0 & 215.0 \\
PNE2 & 203.0 & 254.0 & 250.0 \\
\hline
\end{tabular}

Nota: fuente propia de la investigación. 


\section{Series temporales de algunas esta- ciones de interés}

Otro producto de la combinación de las soluciones libres es la solución final para cada semana procesada. Al tomar esta información y graficarla, se obtiene la serie temporal de cada estación.

Se muestra a continuación, la serie temporal de 4 de las 16 estaciones procesadas en Costa Rica, según el siguiente detalle:

1) En la Gráfica 1 se muestra la serie temporal de la estación SAGE, en San Isidro de El General

2) En la Gráfica 2 se muestra la serie temporal de la estación LIBE, en Liberia
3) En la Gráfica 3 se muestra la serie temporal de la estación NICY, en Nicoya

4) En la Gráfica 4 se muestra la serie temporal de la estación SAJU, en San Juanillo

Para los fines del artículo, se seleccionaron estas estaciones por cuanto dan una idea del efecto de evento del 05 de septiembre de 2012. En la primera estación (SAGE) en la serie temporal no hay evidencia de una discontinuidad asociada al terremoto, mientras que las restantes tres (LIBE, NICY y SAJU) presentan, claramente, una discontinuidad asociada al evento en estudio. Los valores numéricos indicados en rojo en las Gráficas 3, 4 y 5 corresponden a los desplazamientos en los componentes respectivos.

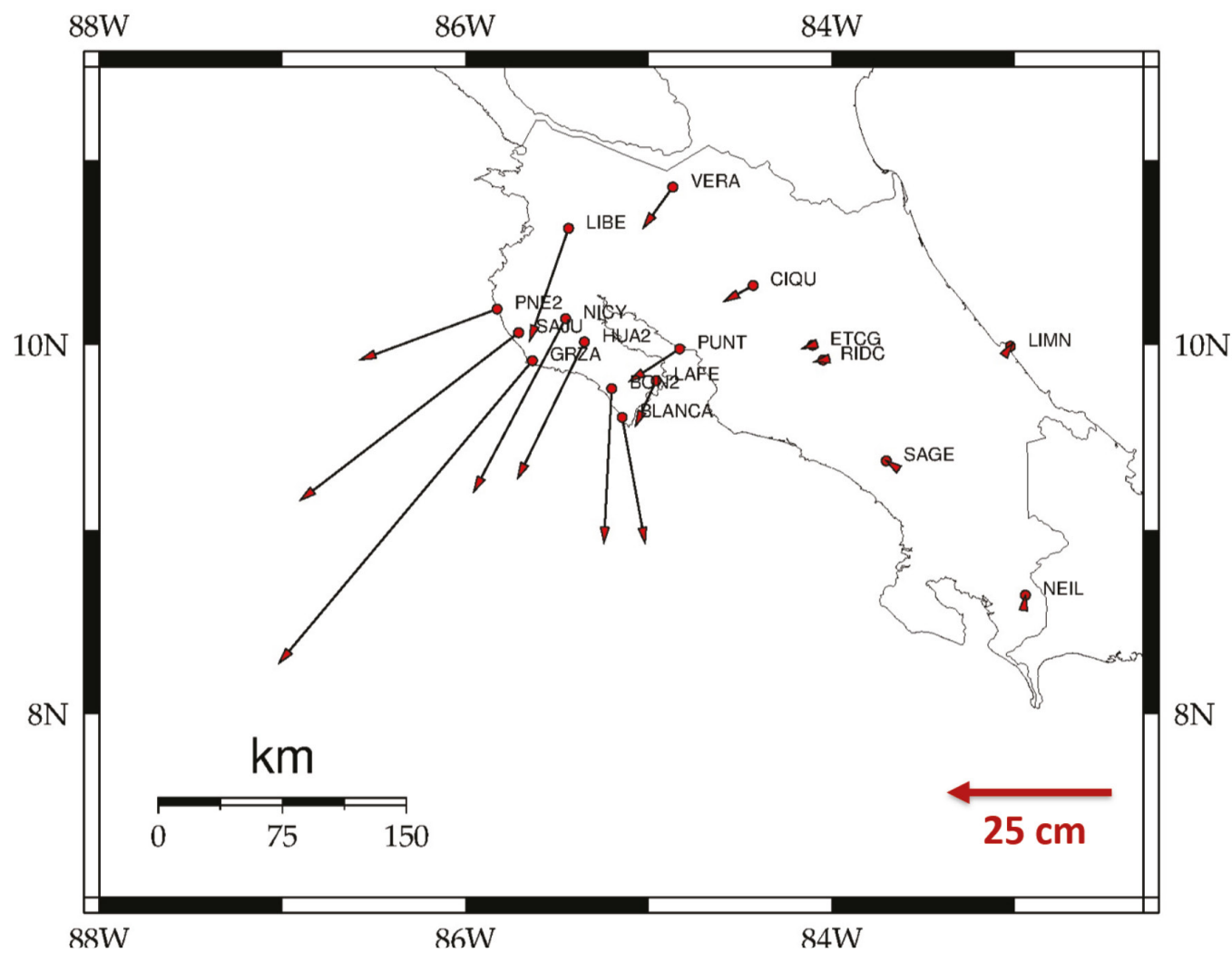

Figura 5. Vectores de desplazamiento horizontal.

Fuente: Elaboración propia, tomando como base la información del programa GMT 

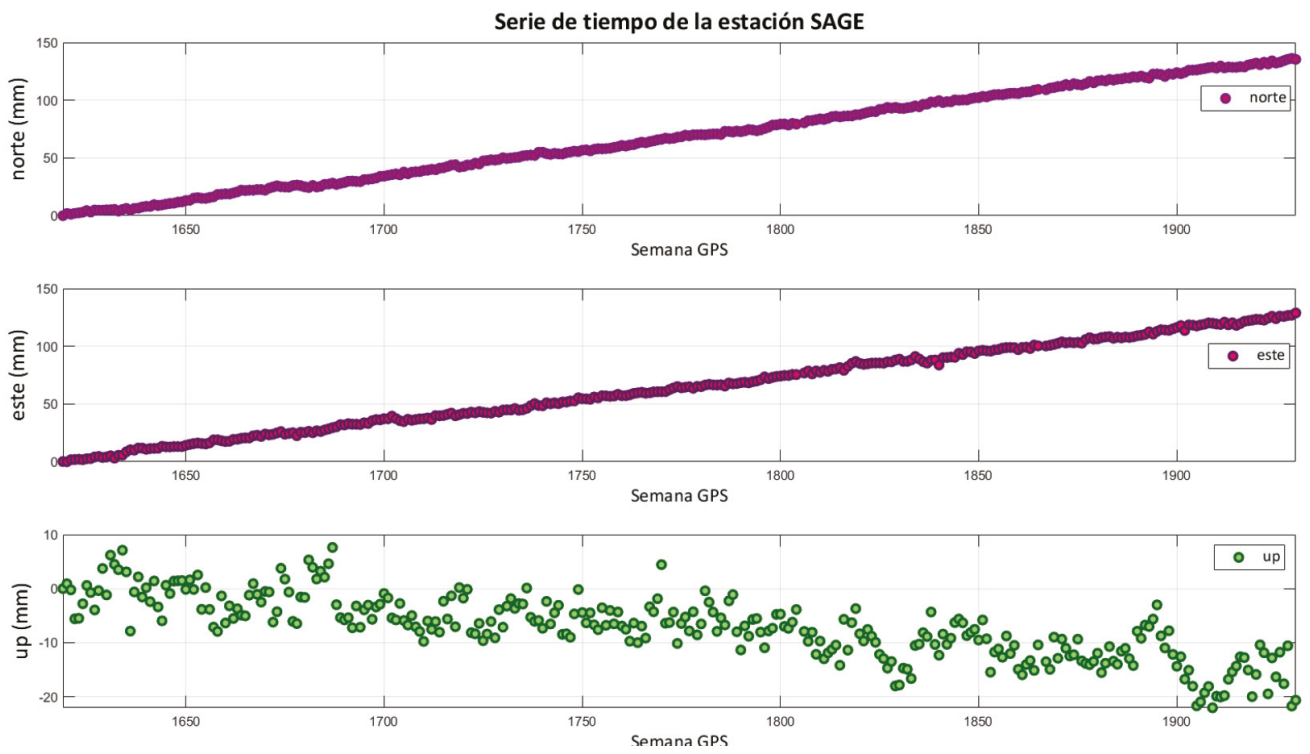

\section{Gráfica 1}

Serie temporal de la estación SAGE

Nota: Fuente propia del estudio.
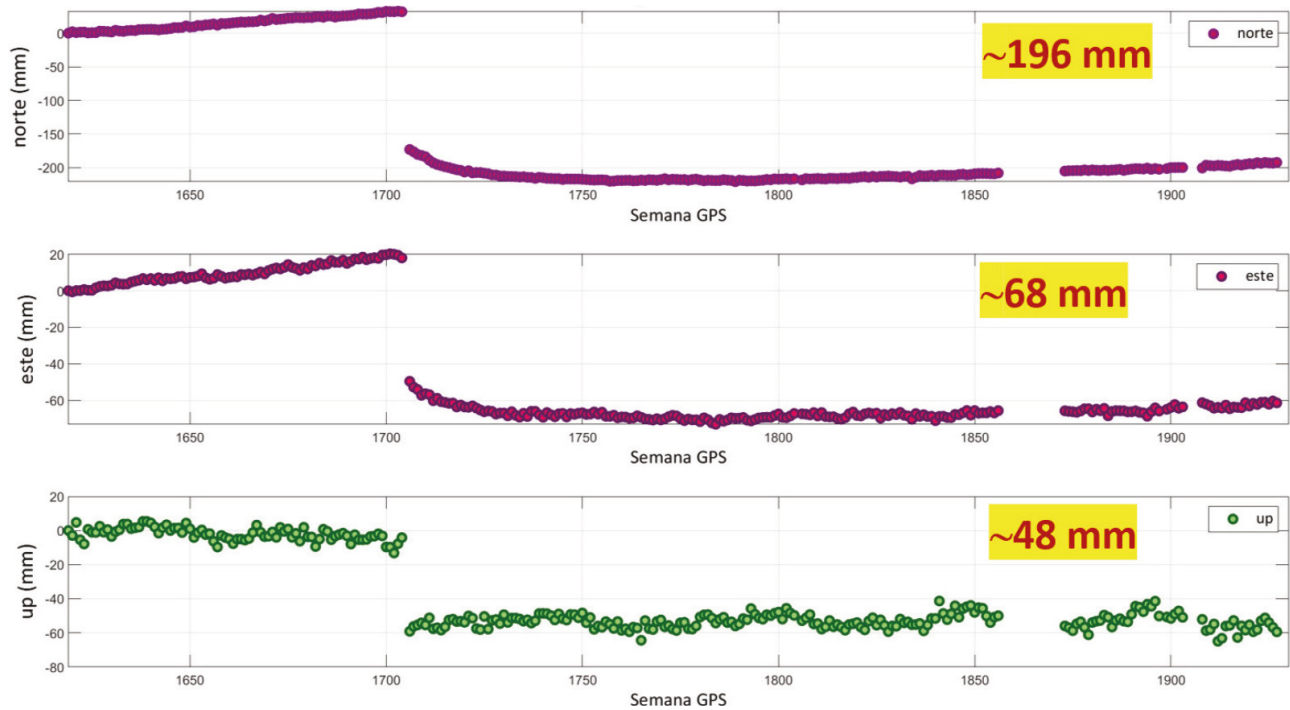

\section{Gráfica 2}

Serie temporal de la estación LIBE

Nota: Fuente propia del estudio. 

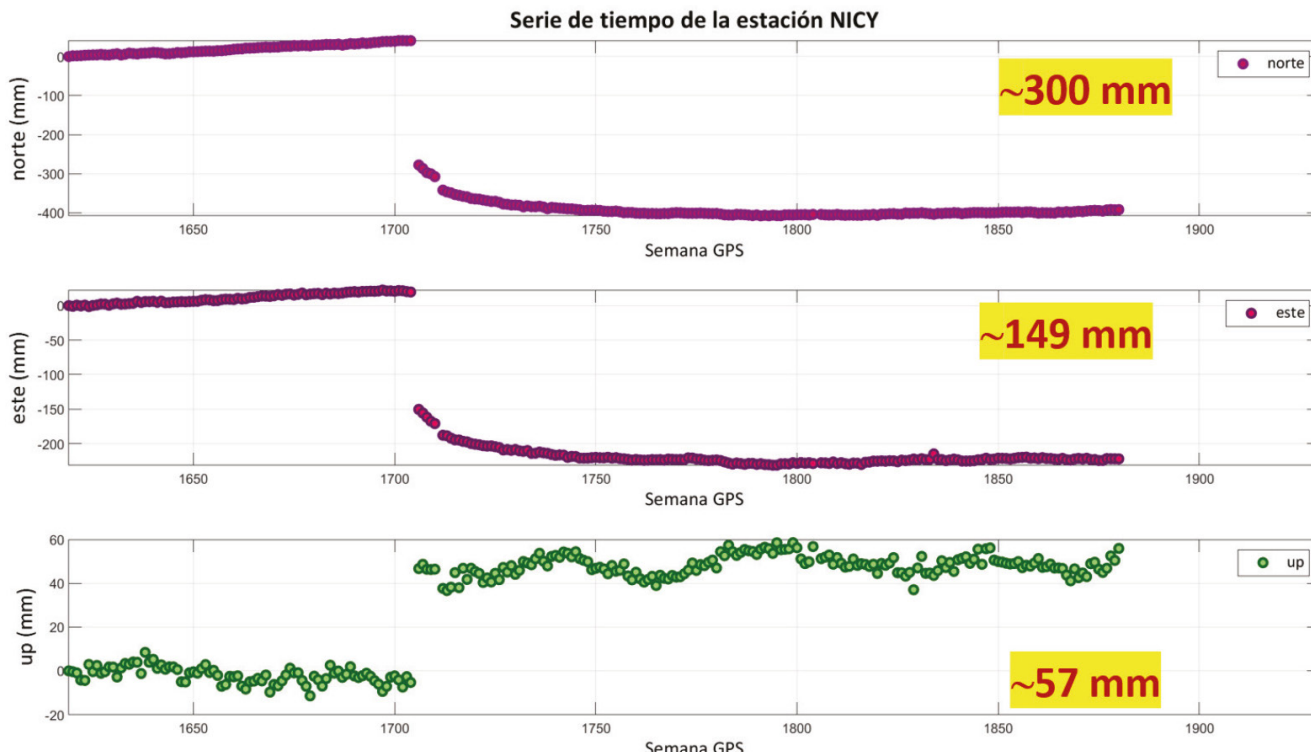

Gráfica 3

Serie temporal de la estación NICY

Nota: Fuente propia del estudio.
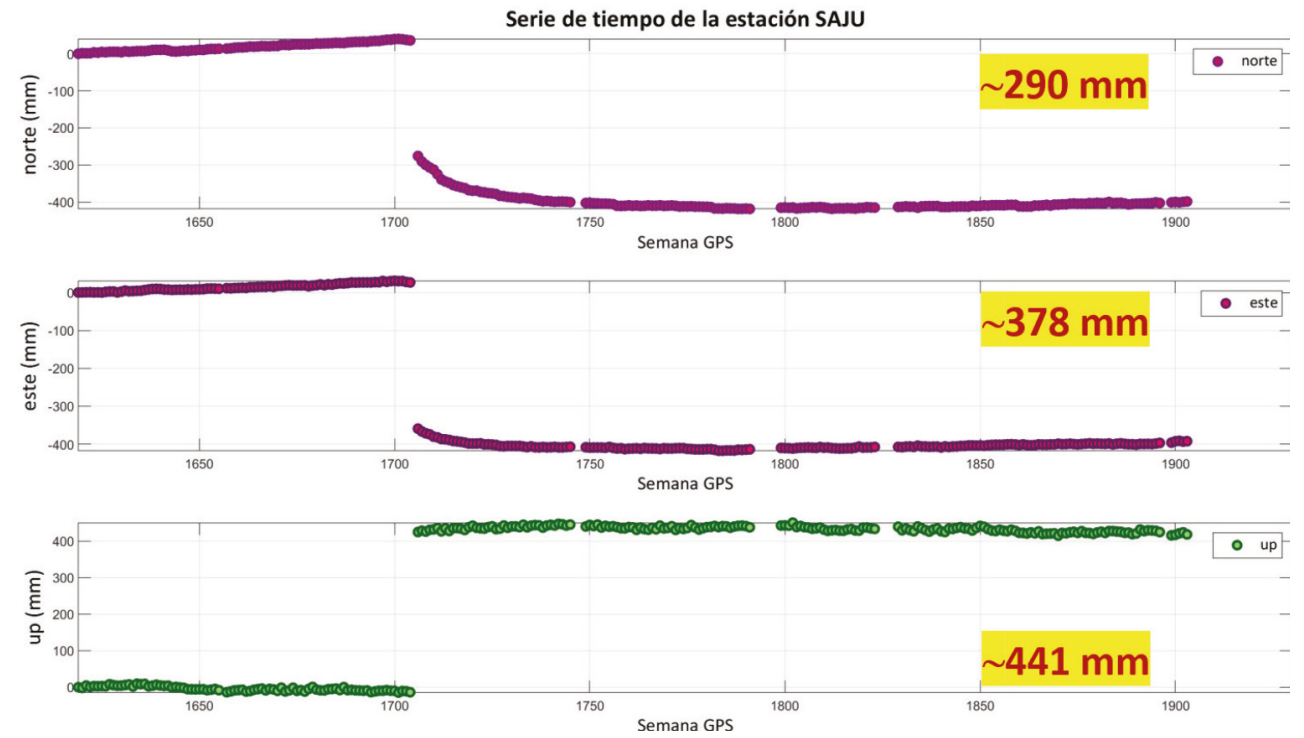

\section{Gráfica 4}

Serie temporal de la estación SAJU

Nota: Fuente propia del estudio. 


\section{Análisis de resultados}

Parte de los resultados obtenidos en el marco del proyecto de investigación fue el cálculo de las coordenadas y las velocidades para un conjunto de estaciones en Costa Rica, así como la generación de las series temporales para cada una de las estaciones de interés.

La Tabla 1 muestra las coordenadas y las velocidades de las 16 estaciones consideradas en el procesamiento válidas para antes del evento, mientras que la Tabla 2 presenta estos valores (coordenadas y velocidades) válidas para épocas después del evento.

Al comparar los valores mostrados en la Tabla 1 y la Tabla 2, y como resultado directo del evento del 05 de septiembre de 2012, se observa como algunas estaciones presentan cambios significativos tanto en su posición (dada por las coordenadas), como en la magnitud de la velocidad y la dirección del movimiento de la estación. Como es de esperarse, en las estaciones más cercanas al epicentro del terremoto es donde se tienen los mayores cambios. Por ejemplo, en la estación SAJU, la magnitud del desplazamiento horizontal es de $47 \mathrm{~cm}$, mientras que el desplazamiento vertical es de $44 \mathrm{~cm}$. En relación con la velocidad, en la coordenada $\mathrm{X}$ antes del evento era de $17.8 \mathrm{~mm} / \mathrm{yr}$, mientras que después del evento el valor es de $0.1 \mathrm{~mm} / \mathrm{yr}$.

En el caso de la estación GRZA, la cual es la estación mas cercana al epicentro considerada en el presente estudio, la magnitud del desplazamiento horizontal es de $68 \mathrm{~cm}, 45 \mathrm{~cm}$ en la componente vertical y en relación con las velocidades, estas eran de $18.7 \mathrm{~mm} / \mathrm{yr}$ en X, $18.7 \mathrm{~mm} / \mathrm{yr}$ en Y y $24.0 \mathrm{~mm} / \mathrm{yr}$ en $\mathrm{Z}$ antes del evento, mientras que los valores para después del evento son $4.2 \mathrm{~mm} / \mathrm{yr}$ en $\mathrm{X},-4.3 \mathrm{~mm} / \mathrm{yr}$ en Y y -6.2 $\mathrm{mm} / \mathrm{yr}$ en $\mathrm{Z}$.
También y como es evidente, las estaciones más alejadas del epicentro son las que presentan las menores variaciones tanto en posición como en velocidad, siendo LIMN, NEIL y SAGE, en Limón, Cuidad Neilly y San Isidro de El General, respectivamente, las estaciones menos afectadas por el evento.

La Grafica 1 muestra la serie temporal de la estación SAGE. Como se desprende del análisis su análisis, no se observa una discontinuidad en la serie, esto debido a que no hay evidencia numérica de que esta estación haya sido afectada por el evento. Lo contrario ocurre con las series de las estaciones LIBE, NICY y SAJU, mostradas en las Gráficas 2, 3 y 4 respectivamente. En estas, se observa claramente una discontinuidad en la serie, asociada al efecto del evento del 05 de septiembre de 2012, siendo de los casos más extremos lo ocurrido en la estación SAJU, donde en el componente vertical es de $44 \mathrm{~cm}$, mientras que en los componentes norte y este la variación es de 29 cm y 37 $\mathrm{cm}$ correspondientemente.

De esta forma, se tiene datos que permiten evidenciar el efecto de los terremotos sobre el marco de referencia de una región o un país, lo que enfatiza la necesidad de estar rutinariamente calculando la posición de las estaciones GNSS que definen el sistema de coordenadas.

\section{Conclusiones}

El presente artículo muestra los resultados tras el procesamiento de 6 años de datos de un conjunto de 16 estaciones GNSS ubicadas en Costa Rica, el cual se se realizó con el fin, principal, de determinar el impacto del terremoto ocurrido el 05 de septiembre de 2012, así como para obtener las series temporales de las estaciones procesadas. El 
periodo comprende del 16 de enero de 2011 al 07 de enero de 2017, para un total de 312 semanas procesadas.

Se estimaron las coordenadas y las velocidades para las estaciones en dos periodos: uno antes del evento (valores válidos para el periodo del 16 de enero de 2011 al 04 de septiembre de 2012) y el otro, para después del evento (valores validos del 06 de septiembre de 2012 al 07 de enero de 2017), así como generar la serie temporal para 16 estaciones costarricenses, las cuales muestran que la deformación asociada al terremoto continuó incluso meses después.

Los resultados obtenidos muestran cómo algunas estaciones no sufrieron cambios por efectos del terremoto (las estaciones ubicadas en Limón y la zona sur del país) mientras que estaciones ubicadas en Guanacaste y Puntarenas sí presentan cambios en su posición y velocidad, asociadas al evento.

Los hallazgos del proyecto permiten evidenciar la necesidad de estar procesando los datos generados por las estaciones GNSS que definen el sistema de coordenadas de una región o un país, de forma continua, máxime cuando estas coordenadas son usadas para generar otros productos que tienen carácter oficial, como cartografía o información catastral. Este procesamiento debe realizarse utilizando herramientas informáticas y modelos (atmosféricos, geofísicos, entre otros) que permitan lograr la mayor exactitud posible en los resultados.

Para el evento estudiado y de acuerdo con los resultados obtenidos, se concluye que coordenadas calculadas para las estaciones GNSS cuya fecha de estimación es anterior al evento del 05 de septiembre de 2012 no son válidas en la actualidad y por tanto, no deben ser usadas para trabajos de índole topográfico o geodésico, por consiguiente, en caso de utilizarlas, se estará introduciendo un error en las coordenadas de puntos nuevos.

En relación con el estado actual del marco de referencia oficial de Costa Rica, el Decreto Ejecutivo 40962-MJP, publicado en el Diario Oficial La Gaceta N. ${ }^{\circ} 66$, del 17 de abril de 2018 oficializa el marco CR14 (Decreto 40962-MJP, 2018) y deja sin efecto marco CR05. La época del marco CR14 es la 2014.59, por lo que estas coordenadas fueron calculadas después del terremoto de 2012. Sin embargo, es necesario que la entidad con la competencia para el fin, establezca las pautas de uso del CR14 y defina una estrategia para trasformar la información del CR05 al CR14.

\section{Agradecimientos}

Trabajo elaborado en el marco del proyecto de investigación código SIA 000815: Estudio de deformaciones cosísmicas y postsísmicas en Costa Rica como una contribución al mantenimiento del marco geodésico del país.

Se agradece al Sr. Jean Carlo Arroyo Ruiz y el Máster Carlos Sevilla Hernández, estudiante y docente de la ETCG, respectivamente, quienes programaron la rutina para extraer las coordenadas del archivo con las soluciones que son la base para la preparación de las series temporales y a la Ing. Diana Paniagua Jiménez, quien realizó valiosos aportes a la versión final del artículo.

\section{Referencias}

Altamimi, Z.; Collilieux, X. y Métivier, L. (2011). ITRF2008: an improved solution of the international terrestrial reference frame. Journal of Geodesy, 85(8), 457-473. doi: 10.1007/ s00190-011-0444-4 
Angermann, D.; Drewes, H.; Krügel, M.; Meisel, B.; Gerstl, M.; Kelm, R.; Müller, H.; Seemüller, W. y Tesmer, V. (2004). ITRS Combination Center at DGFI: A Terrestrial Reference Frame Realization. Recuperado de http://www. dgk.badw.de/fileadmin/docs/b-313.pdf

Bevis, M., \& Brown, A. (2014). Trajectory models and reference frames for crustal motion geodesy. Journal of Geodesy, 88(3), 283-311. doi: 10.1007/s00190-013-0685-5

Bloßfeld, M., Seitz, M., \& Angermann, D. (2014). Non-linear station motions in epoch and multi-year reference frames. Journal of Geodesy, 88(1), 45-63. doi: 10.1007/ s00190-013-0668-6

Brockmann, E. (1997). Combination of Solutions for Geodetic and Geodynamic Applications of the Global Positioning System (GPS). Zürich, Institut für Geodäsie und Photogrammetrie, Schweizerischen Geodätischen Kommission. Vol. 55

Bruni, S.; Zerbini, S.; Raicich, F.; Errico, M. y Santi, E. (2014). Detecting discontinuities in GNSS coordinate time series with STARS: case study, the Bologna and Medicine GPS sites. Journal of Geodesy, 88(12), 1203-1214. doi: 10.1007/s00190-014-0754-4

Decreto Ejecutivo 40962-MJP. (2018). Actualización del sistema geodésico de referencia horizontal oficial para Costa Rica. Diario Oficial La Gaceta $N^{\circ} 66$ del 17 de abril de 2018.

Hofmann-Wellenhof, B. y Moritz, H. (2005). Physical Geodesy. New York: Springer

Ostini, L. (2012). Analysis and Quality Assessment of GNSS-Derived Parameter Time Series. Tesis para obtener el titular de Doctor en Astronomía, Astronomical Institute, University of Bern, Bern, Switzerland.

Petit, G. y Luzum, B. (Eds.). (2010). IERS Conventions. Germany: Verlag dews Bundesamtes für kartographie und Geodäsie.
Programa de Regularización de Catastro y Registro de Costa Rica. (2007). El sistema de referencia CR05 y la proyección Transversal Mercator para Costa Rica. Costa Rica.

Ramírez, M. y Vargas, E. (2014). Análisis de calidad del mapa catastral en Costa Rica utilizando Sistemas de Información Geográfica (SIG) de licencia pública. Revista geográfica de América Central, (53), 173-188. doi: 10.15359/ rgac. $2-53.8$

Sánchez, L. y Drewes, H. (2016). SIR15P01: Multiyear solution for the SIRGAS Reference Frame, link to ZIP archive. PANGAEA. doi: 10.1594/PANGAEA.862536

Seeber, G. (2003). Satellite Geodesy. Berlin, Alemania: Walter de Gruyter. doi: $10.1515 / 9783110200089$

Seitz, M. (2014). Comparison of different combination strategies applied for the computation of terrestrial reference frames and geodetic parameter series. In: Kutterer H., Seitz F., Alkhatib H., Schmidt M. (Eds.) The 1st International Workshop on the Quality of Geodetic Observation and Monitoring Systems (QuGOMS'11), IAG Symposia 140: 57-64. doi: 10.1007/978-3-319-10828-5_9

Teunissen, P. J. G. (1998). Quality Control and GPS. Faculty if Civil Engineering and Geosciences, section MGP. Thijsseweg 11, 2629 JA Delft, The Netherlands (DOC 19)

Thaller, D. (2008). Inter-technique combination based on homogeneous normal equation systems including station coordinates, Earth orientation and troposphere parameters. Scientific Technical Report STR08/15

Torge, W. (2001). Geodesy. Berlin, Alemania: Walter de Gruyter. doi: 10.1515/9783110879957

Williams, S. D. P. (2008). CATS: GPS coordinate time series analysis software. GPS Solutions, 12(2), 147-153. doi: 10.1007/ s10291-007-0086-4

\section{(c) $\odot \Theta \Theta$}

Estudio del efecto de un terremoto sobre un marco geodésico de referencia (Jose Valverde-Calderón) por Revista Uniciencia se encuentra bajo una Licencia CreativeCommons Atribución-NoComercial-SinDerivadas 3.0 Unported. 